\title{
Experimental Tests of the Standard Model of Weak Interactions
}

\author{
Dalibor Zakoucky ${ }^{1}$ \\ Nuclear Physics Institute AS CR \\ 25068 Rez near Prague, Czech Republic \\ E-mail: zakoucky@ujf.cas.cz
}

\section{WITCH and ISOLDE collaborations}

CERN

Geneve, Switzerland

E-mail:

Weak interactions are described by the Standard Model which postulates the basic assumption about the pure "V(ector)-A(xial vector)" character of the interaction. Our experimental project at ISOLDE, CERN tries to probe the properties of weak interactions in order to look for the forbidden (scalar, tensor) components or at least significantly improve their current experimental limits.

XLVIII International Winter Meeting on Nuclear Physics - BORMIO2010

Bormio, Italy

January 25-29 2010

\footnotetext{
${ }^{1}$ Dalibor Zakoucky
} 


\section{Motivation}

The Standard Model as a very successful theory of Electroweak Interactions is believed to be only the "low-energy" approximation of a more fundamental theory since it is based on a number of basic assumptions. It contains too many parameters that have to be determined by experiment while also some features such as e.g. parity violation and $\mathrm{CP}$-violation are not explained by the model itself. Moreover with the discovery of neutrino oscillations [1] the existence of new physics not included in the Standard Model was already demonstrated.

One of the basic ingredients of the Standard Model is the assumption about the pure "V-A" (Vextor-AxialVector) character of the interaction while the other possible types of interaction (scalar, tensor) are excluded. Nevertheless, even after half a century of development of the model and experimental testing of its fundamental ingredients, experimental limits for admixtures of scalar and/or tensor interactions are still as high as $\sim 8 \%$ [2 ]. So the way to extend the Standard Model in order to build a more fundamental theory is to look for the deviations from the Standard Model predictions or to check its basic assumptions as e.g. the pure V-A character of the interaction. This can be done either in the domain of nuclear $\beta$-decay where fundamental properties of the weak interaction, such as parity violation and the V-A structure of the interaction, were originally discovered - or at the highest energies in particle accelerators or colliders where new particles mediating the forbidden types of interactions are looked for. Experimental efforts in both domains are complementary in a sense that from one side the new precision measurements in nuclear $\beta$-decay are carried out to determine the parameters related to the structure of the weak lepton-quark current or to search for tiny effects that would be induced by the exchange of new massive particles and from the other side the experiments at high-energy colliders search for the direct production of these new massive particles and study their properties.

\section{Experiment WITCH}

\subsection{Experimental principle}

Experimental study of the weak interaction properties can be done by studying special correlations between the participants in the $\beta$-decay. The correlation between the spin of $\beta$ decaying nucleus and momentum of emitted $\beta$-particle is sensitive to new time reversal invariant tensor component in the weak interaction which can be studied by the precision measurement of the $\beta$-asymmetry parameter in nuclear $\beta$-decay (e.g. by the measurement of the angular distribution of $\beta$-particles emitted by the oriented samples). Another correlation in $\beta$ decay sensitive to the new physics outside of Standard Model is a $\beta-v$ correlation. Due to the difficulty to detect neutrinos this can be replaced by the study of daughter nuclei recoiling after the $\beta$-decay. Of course, due to the low energy of recoiling nuclei $(\sim \mathrm{eV})$, the standard radioactive samples cannot be used for this purpose since recoiling nuclei will not be able to escape from the sample. The solution for this problem is provided by ion traps which are able to produce isotopically pure, very well localised samples with small density which are scattering and absorption free. 
Since the shape of the energy spectrum of recoiling nuclei is very sensitive to the character of the interaction (Fig.1) the precise measurement of the shape of recoil spectrum can distinguish between contributions of different modes and reveal even a small admixture of scalar component ((forbidden in the Standard Model) in the dominant (allowed) vector mode.

For this purpose a new online facility WITCH (Weak Interaction Trap for CHarged particles) for the measurements of the $\beta-v$ angular correlation in the nuclear $\beta$-decay has been built at ISOLDE separator in CERN [3]. This world unique combination of 2 Penning traps and retardation spectrometer allows us to trap radioactive ions produced by ISOLDE separator, cool them, let them decay and probe the energy spectrum of recoiling nuclei by the retardation spectrometer.

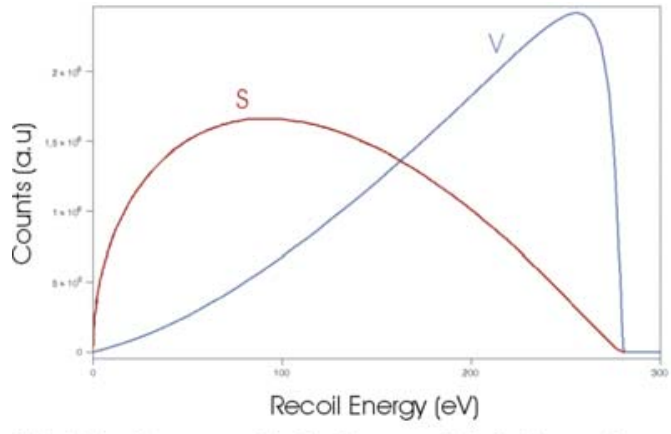

Fig.1 Recoil spectrum for the Vector and Scalar interactions

\subsection{Experimental setup}

The main components of WITCH setup (Fig. 2) are two Penning ion traps and a retardation spectrometer. Setup is installed on the beamline of the ISOLDE separator which provides the nuclei to be studied. Isotopes of interest are produced in the ISOLDE target/ion source unit by bombarding thick targets with the intensive proton beam $(\sim 1 \mu \mathrm{A})$ with the energy $\sim 1 \mathrm{GeV}$ from PS Booster accelerator and the nuclei produced are extracted and separated by the ISOLDE separator. Beam is stopped, cooled and bunched in the Penning trap REXTRAP and then with the extraction energy $30 \mathrm{keV}$ transferred to WITCH. Here is the beam bent vertically upwards and then decelerated by a combination of electrodes and a pulsed drift tube. Ions are then trapped and cooled with buffer gas in the first Penning ion trap (cooler trap) and then transferred to the second (decay) trap. In the cooler trap not only are the ions cooled but also the ion bunch can be cleaned of possible contaminants. After sufficient cooling the ion cloud is transferred to the decay trap which is well pumped to high vacuum. The recoiling daughter ions after the $\beta$-decay in this trap leave the ion cloud and the trap potential without any significant energy loss or scattering and they are guided by a $9 \mathrm{~T}$ magnetic field into the retardation spectrometer (placed in a $0.1 \mathrm{~T}$ magnetic field) where their energy is scanned

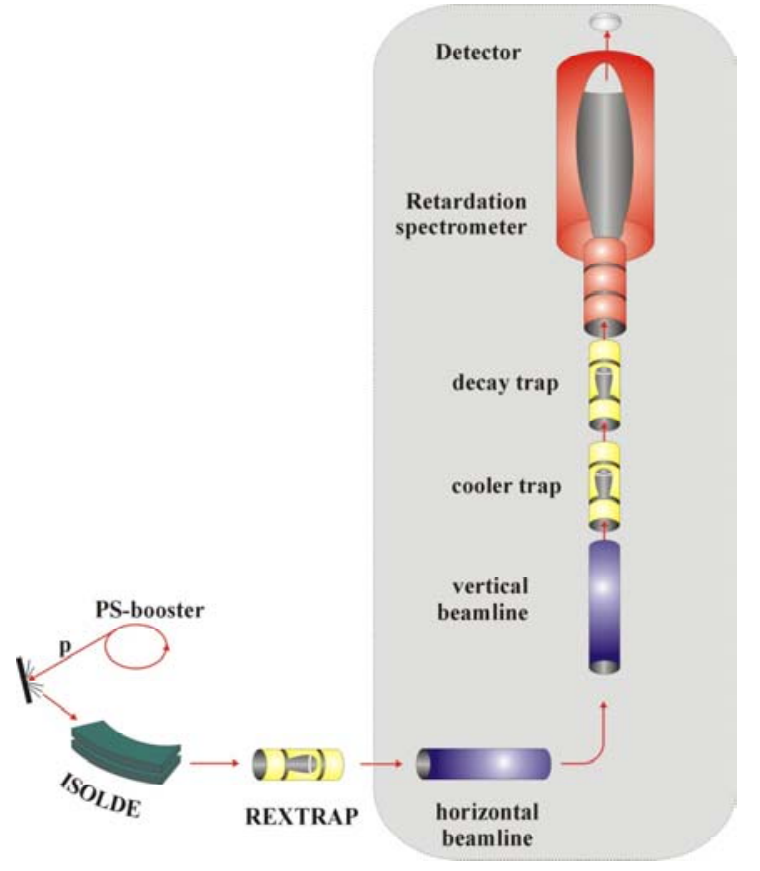

Fig.2: WITCH setup at ISOLDE,CERN 
by changing the height of an electrostatic barrier. On top of the setup a $4 \mathrm{~cm}$ diameter position sensitive MCP detector is placed for the detection of the recoil ions that pass through the retardation spectrometer. In this way the energy spectrum of recoil ions from $\beta$-decay can be measured for a wide variety of isotopes, independent of their specific properties.

\subsection{Experimental results and status}

Experiment WITCH was built in the ISOLDE hall in CERN during 2004 and then gradually tested and optimised and brought into operation. In November 2006 a first complete experiment with the WITCH setup was performed with the isotope ${ }^{124} \mathrm{In}$, successfully demonstrating the proof-of-principle of the experiment [4]. After this experiment first physics run in 2007 was performed with the isotope ${ }^{35} \mathrm{Ar}$ which was the best candidate for a measurement testing scalar component of weak interactions. This run failed due to several reasons. The ISOLDE beam with mass $A=35$ contained unexpectedly 400 times more stable ${ }^{35} \mathrm{Cl}$ then requested ${ }^{35} \mathrm{Ar}$ and therefore the traps were almost fully filled with the unwanted isotope. Even after optimization the ratio ${ }^{35} \mathrm{Ar} /{ }^{35} \mathrm{Cl}$ went only to $1: 25$ but with the greatly reduced yield. Secondly the ions were in WITCH (and also in REXTRAP) mostly lost due to the high rate of charge exchange which caused that the ions were neutralized and therefore lost for further manipulation (the charge exchange half-life was of the order of $\sim 10$ ms while the time needed for cooling was $\sim 100 \mathrm{~ms}$ ). Also a high rate of secondary ionization was observed which caused too high background. As a result of these investigations, the reasons for the problems were identified and a complete reconstruction of WITCH apparatus was done in period 2007-2008.

In order to get rid of the charge exchange the vacuum in the setup and overall cleanness of the system had to be improved - from existing $\sim 10^{-8}$ mbar to $\sim 10^{-9}$ mbar in the Penning trap regions and in the spectrometer. The pumping capacity was increased by installation of additional pumps and NEG (Non Evaporable Getter) coatings were applied on the inside walls of chambers, additional NEG foils with resistive heaters were installed around the traps. Apart from that the general "purity" of the system was improved. Whole buffer gas system was replaced to be "all metal" in order to get rid of contaminants in the buffer gas which would neutralize rather then cool the ions in cooler trap. Moreover, it turned out that the requirement of ultrahigh vacuum is not compatible with the usage of teflon components inside of the system. Therefore all teflon and kapton coated wires were replaced by the ceramics. To reduce the problem with secondary ionisation the whole system of electrodes was redesigned - new electrodes were made from titanium instead of aluminum, all the edges were rounded and electrodes were cleaned and (electro) polished in order to remove sparking .

In 2009 new experiment with ${ }^{35} \mathrm{Ar}$ was performed which proved that the reconstruction was successful and the most of the problems experienced in the previous run was solved. General vacuum in WITCH reached $\sim 10^{-9}$ mbar and charge exchange problem was solved (charge exchange rate is now only $10 \%$ for a trapping time of $500 \mathrm{~ms}$ in the cooler trap). Also the secondary ionisation problem was reduced. Due to the developments on the ISOLDE separator side, beam composition was significantly improved reaching the ratio ${ }^{35} \mathrm{Ar} /{ }^{35} \mathrm{Cl}$ of 1:5. 
Summarising the results of reconstruction, the overall efficiency of the setup has been improved by a factor of $\sim 60$, mass dependent purification of the ion beam was achieved for the first time, we observed recoil ions of ${ }^{35} \mathrm{Ar}$. Full data analysis is still ongoing and systematic effects are under investigation.

\section{Conclusions and outlook}

In 2010 we plan to measure the response function of the entire system with the isotope ${ }^{144} \mathrm{Eu}$ which decays by $\beta^{+}$-decay and also by EC (electron capture). The mono-energetic recoils from the EC decays will enable us to calibrate full system and measure the response function. After that the experimental setup WITCH is ready to accomplish its main goal - to perform real precision measurement of the recoil spectra - first for the most promising isotope ${ }^{35} \mathrm{Ar}$. We plan to obtain good statistics for the recoil spectrum and be able to determine with good accuracy the correlation parameter $\boldsymbol{a}$ which will enable us to improve the current limits for the existence of the scalar component of weak interactions.

Apart from the main goal of the WITCH experiment, there are still many additional interesting observables which can be studied at WITCH and which are in planning. Available weak interaction phenomena include search for possible tensor component of weak interactions, measuring Fermi/Gamow-Teller mixing ratios for $\beta$-decays, ElectronCapture/ $\beta^{+}$ratios, search for possible heavy neutrinos by studying precisely the shape of the recoil spectrum and looking for kinks,...

Another whole broad field opened for WITCH is the "trap assisted spectroscopy" which could be performed after replacing the top MCP detector by the tape station. This would use the unique performance of WITCH to purify the beam and get very pure samples of short lived exotic nuclei available for further studies in the fields of nuclear and possibly also atomic physics.

This work was supported by the Grants of the Ministry of Education of the Czech Republic 1P04LA211 and LA08015.

\section{References}

[1] R.D. McKeown and P. Vogel, Neutrino masses and oscillations: triumphs and challenges, Phys. Rep. 394 (2004) 315

[2] N. Severijns, M. Beck, O. Naviliat-Cuncic, Tests of the standard electroweak model in nuclear beta decay, Rev. Mod. Phys. 78 (2006) 991

[3] N.Severijns et al., Search for New Physics in Beta-Neutrino Correlations using Trapped Ions and a Retardation Spectrometer, CERN-INTC-2005-014 ; CERN-INTC-2008-004

[4] V.Yu. Kozlov, M. Beck, S. Coeck, P. Delahaye, P. Friedag, M. Herbane, A. Herlert, I.S. Kraev, M. Tandecki, S. Van Gorp, F. Wauters, Ch. Weinheimer, F. Wenander, D. Zákoucký, N. Severijns; The WITCH experiment: Acquiring the first recoil ion spectrum; Nuclear Instr. and Methods in Physics Research B 266 (2008) 4515 